\title{
Chemical doping of individual semiconducting carbon-nanotube ropes
}

\author{
Marc Bockrath, J. Hone, A. Zettl, and Paul L. McEuen \\ Department of Physics, University of California, Berkeley, California 94720 \\ and Materials Science Division, Lawrence Berkeley National Laboratory, Berkeley, California, 94720 \\ Andrew G. Rinzler and Richard E. Smalley \\ Center for Nanoscale Science and Technology, Rice Quantum Institute, \\ and Department of Chemistry and Physics, MS-100, Rice University, P.O. Box 1892, Houston, Texas 77251
}

(Received 1 November 1999)

\begin{abstract}
We report the effects of potassium doping on the conductance of individual semiconducting single-walled carbon nanotube ropes. We are able to control the level of doping by reversibly intercalating and deintercalating potassium. Potassium doping changes the carriers in the ropes from holes to electrons. Typical values for the carrier density are found to be $\sim 100-1000$ electrons $/ \mu \mathrm{m}$. The effective mobility for the electrons is $\mu_{\mathrm{eff}} \sim 20-60 \mathrm{~cm}^{2} \mathrm{~V}^{-1} \mathrm{~s}^{-1}$, a value similar to that reported for the hole effective mobility in nanotubes [R. Martel et al., Appl. Phys. Lett. 73, 2447 (1998)].
\end{abstract}

Recently, much attention has been focused on the transport properties of $\sim 1-2 \mathrm{~nm}$ diam single-walled carbon nanotubes (SWNT's). The band structure of a given SWNT depends on its chirality and is either a one-dimensional (1D) metal or a 1D semiconductor with a band gap on the order of $0.6 \mathrm{eV} .^{2,3}$ Recent work has shown that semiconducting nanotubes can exhibit transistor action that is similar in operation to a MOSFET. ${ }^{1,4}$ In these experiments, a gate electrode is used to electrostatically vary the charge density in the nanotubes. The dependence of the conductance on the carrier density indicates that the carriers added to the tubes are holes.

Besides using a gate, another way to change the charge density in a semiconductor is by chemical doping. Previous work on mats of SWNT's has shown that potassium acts as an electron donor to the nanotubes ${ }^{5}$ and that their conductance is increased significantly by doping with potassium vapor. ${ }^{6,7}$ However, the interpretation of these experiments is complicated by the fact that mats consist of both semiconducting and metallic nanotubes, and that transport through the mats includes hopping between nanotubes and/or bundles.

Here we report the controlled chemical doping of individual semiconducting nanotube ropes with potassium. This doping changes the carriers in the ropes from holes to electrons. We estimate the number of carriers added and hence the number of intercalated potassium atoms. In addition we determine an effective mobility $\mu_{\text {eff }}$ for the electrons, which is found to be similar in magnitude to the value reported for holes. ${ }^{1}$

The samples are made by placing ropes on top of $\mathrm{Au}$ electrodes that have $500 \mathrm{~nm}$ separation ${ }^{8}$ as shown in the inset of Fig. 1. These electrodes are on top of a degenerately doped oxidized Si wafer. ${ }^{9}$ The degenerately doped substrate is used as a gate. Once the samples are made, semiconducting and metallic devices are distinguished by their room temperature conductance behavior with respect to gate voltage. The conductance of metallic tubes shows relatively little dependence on gate voltage, whereas the conductance of semiconducting nanotubes shows a very strong dependence. ${ }^{4,10}$
An example of semiconducting behavior is shown in Fig. 2 . The open circles in Fig. 2 show the conductance of a $3 \mathrm{~nm}$ diam rope as a function of gate voltage. At positive gate voltages, the conductance of the rope approaches zero. As a negative gate voltage is applied, the conductance begins to increase as holes are added to the rope. ${ }^{4}$ This increase in conductance is approximately linear in gate voltage. A similar slope has been found in all the samples we have studied to within a factor of 3 .

Once we have obtained samples that show the above semiconducting behavior, we dope them with potassium. To do this, we place the sample in a glass doping vessel, which consists of a glass tube with wire feedthroughs, as shown in the inset of Fig. 1. The vessel is first attached to a pump and heated in vacuum at $200{ }^{\circ} \mathrm{C}$ to remove moisture and other

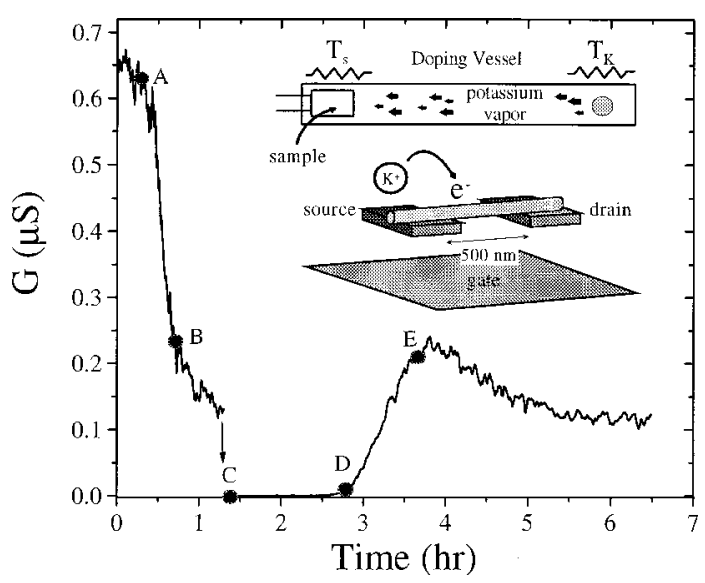

FIG. 1. Main panel: Conductance of potassium-doped semiconducting sample vs time. At point $A$ heat was applied to the sample to de-intercalate potassium from the doped sample. At point $B$ heating was stopped temporarily. At point $C$ further heating resulted in the sample becoming insulating. Heat was then applied to the potassium. At point $D$, the vapor pressure of the potassium is sufficient to begin doping the sample. At point $E$, heating is stopped and the entire vessel begins to return to room temperature. Inset: diagram of doping vessel and device geometry. 


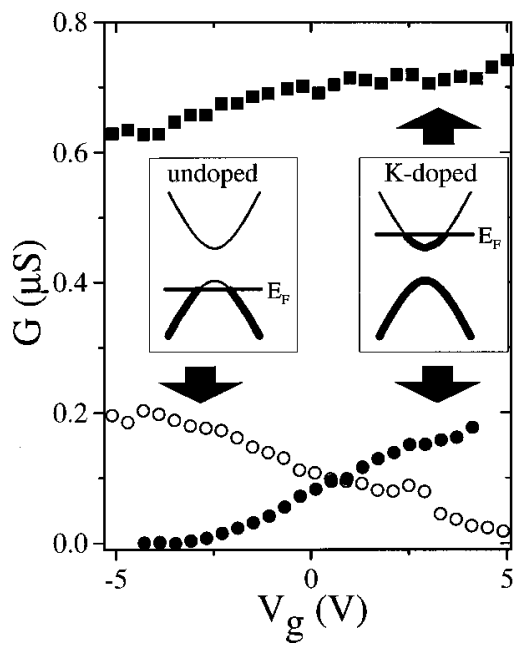

FIG. 2. Open circles: Conductance vs gate voltage for an undoped nanotube rope. The conductance increases with decreasing gate voltage, indicating $p$-type behavior. The left inset shows a schematic band-structure diagram corresponding to this situation. Filled squares: Conductance of a different sample after doping with potassium. Filled circles: same sample after a cycle of deintercalation and re-intercalation at a lower doping level. The conductance increases with increasing gate voltage, indicating $n$-type behavior. The right inset shows a schematic band-structure diagram corresponding to this situation.

volatile impurities. It is then transferred to an inert atmosphere glove box, where a small amount of potassium is placed into the tube. Finally, the vessel is removed from the glove box, pumped out, and sealed with a torch. Doping is accomplished by differentially heating the two ends of the vessel to temperatures $T_{s}$ (for the sample end) and $T_{K}$ (for the potassium end). We have performed doping experiments on several samples; all yielded similar results. However, all of the data shown here are taken from s single sample.

While heating the sample in vacuum, we find that the conductance of undoped semiconducting nanotubes decreases dramatically until they become insulating. Although we do not fully understand this behavior, we have considered two possibilities. One is that the semiconducting nanotubes become insulating as a result of changes in the local electrostatic environment of the nanotube (e.g., neutralization of trapped oxide charge). Another possibility is that they may be doped by molecular species that are adsorbed from the air, which are removed by heating in vacuum. A more complete understanding of this will require further work.

Following the bake-out, we have found that heating the potassium briefly with a flame causes the conductance of semiconducting nanotubes to increase from zero to a finite value. Since the potassium donates electrons to the nanotubes one expects to be able to observe $n$-type behavior in the doped nanotubes. Indeed, we find that this is the case, as shown in Fig. 2. The data plotted with filled squares in Fig. 2 show the conductance of the doped rope as a function of gate voltage after exposure to potassium vapor. Unlike what is observed for intrinsic nanotubes, the conductance of the doped nanotubes increases with increasing gate voltage. This implies that the carriers are electrons, rather than holes. ${ }^{11}$

We have found that this doping is reversible. The main panel of Fig. 1 shows the conductance of a semiconducting sample after the initial potassium doping. No gate voltage is applied, unless otherwise noted. At point $A$, the conductance is relatively stable at a value of $\approx 0.6 \mu \mathrm{S}$. At this point, we applied heat to the sample $\left(T_{s} \sim 400 \mathrm{~K}\right)$. As time evolves, the sample becomes less conductive as the potassium is deintercalated and electrons are removed. ${ }^{6,7}$ We stopped heating the sample at point $B$, at which time the conductance begins to stabilize at a value of $\sim 0.1 \mu \mathrm{S}$. Further heating caused the sample to become insulating (not shown), implying that the majority of the intercalated potassium has been removed.

To add potassium to the sample in a controlled way, we heated both the sample and the potassium simultaneously using the heaters. Throughout this procedure, the sample was maintained at a slightly higher temperature than the potassium $\left(T_{s}-T_{K} \sim 20 \mathrm{~K}\right)$ to avoid gross deposition of potassium on the sample. At point $C, T_{K}=400 \mathrm{~K}$, while $T_{s}=420 \mathrm{~K}$. At this point, the vapor pressure of the potassium is insufficient to dope the sample, and it remains insulating. At point $D$, $T_{K}=450 \mathrm{~K}$, and the vapor pressure of the potassium becomes large enough to begin doping the sample. As time evolves, the conductance increases. At point $E$ both the sample and the potassium are allowed to cool to room temperature. Upon cooling down from point $E$, the conductance decreases until the temperature stabilizes at room temperature, and the conductance stabilizes at $G \sim 0.12 \mu \mathrm{S}$. The filled circles in Fig. 2 show the conductance of the sample as a function of gate voltage after this procedure. At negative gate voltages, the sample is insulating. At a threshold voltage $V_{\text {th }} \sim-3.8 \mathrm{~V}$, the conductance begins to increase, rising approximately linearly with gate voltage.

We now discuss these results. First, we estimate the number of potassium atoms added to the sample. When the gate voltage is zero, the charge density in the ropes is determined by their local electrostatic environment, which includes the ionized potassium donors. If we make the assumption that when the device is off that the electrons in the rope are depleted, then the electron density $n_{e}$ at $V_{g}=0$ will be given by $e n_{e}=C_{L}\left|V_{\text {th }}\right|$. Here, $C_{L}$ is the capacitance per unit length of the rope to the gate electrode and $V_{\text {th }}$ is the threshold voltage at which the device conducts. Measurements of metallic nanotubes in a similar geometry in the Coulomb blockade regime give a typical value $C_{L} \sim 20 e^{-} / \mathrm{V} \mu \mathrm{m}=3.2$ $\times 10^{-12} \mathrm{~F} / \mathrm{m}$. ${ }^{9}$ With the approximation that at $V_{g}=0$ each potassium atom donates an electron to the rope, we have $n_{K} \sim n_{e}$. Thus from the data shown in Fig. 2 as filled circles we conclude that $n_{K} \sim 100 \mu \mathrm{m}^{-1}$. For the higher conductance data (filled squares) we estimate that the number of carriers is $n_{K} \sim 700 \mu \mathrm{m}^{-1}$ from the ratio of the conductance between the two doping levels at $V_{g}=0$. These doping levels correspond to a few potassium atoms per 1000 carbon atoms. In comparison to the $\mathrm{KC}_{8}$ stoichiometric doping levels reported by Lee $e t$ al. and Grigorian et al., ${ }^{6,7}$ this is therefore, very light doping.

We note that similar to the case of $p$-type semiconducting nanotubes, ${ }^{1}$ the increase in conductance is approximately linear in gate voltage. We can thus determine an effective mobility for the carriers: $\mu_{\mathrm{eff}}=L^{2} / C d G / d V_{g}$, where $L$ is the length of the sample, $C$ is the capacitance, $G$ is the conductance, and $V_{g}$ is the gate voltage. For the undoped sample in 
Fig. 2 we find that the hole mobility is given by $\mu_{\text {eff }} \sim 60$ $\mathrm{cm}^{2} \mathrm{~V}^{-1} \mathrm{~s}^{-1}$ in agreement with values reported by others. ${ }^{1}$ For the doped sample, the higher conductance data in Fig. 2 (solid squares) yield $\mu_{\text {eff }} \sim 20 \mathrm{~cm}^{2} \mathrm{~V}^{-1} \mathrm{~s}^{-1}$, while the lower conductance data (filled circles) yield $\mu_{\mathrm{eff}} \sim 60 \mathrm{~cm}^{2} \mathrm{~V}^{-1} \mathrm{~s}^{-1}$. These values for the electron effective mobility are thus similar to typical values for the hole effective mobility. This indicates that the scattering mechanism for electrons and holes is likely similar.

In conclusion, we report the chemical doping of individual semiconducting nanotube ropes with potassium. We find that this chemical doping changes their conductance from zero to a value on the order of $1 \mu \mathrm{S}$. From the gate voltage dependence of the conductance, we deduce that the charge carriers are electrons. This is explained by a model in which the potassium donates electrons to the nanotube rope, resulting in mobile charge carriers at the Fermi level. The effective mobility for the electrons is similar in magnitude to the effective mobility of holes. These experiments open the way toward other experiments that require controlled doping, such as making nanoscale $p-n$ junctions. ${ }^{12}$

We would like to thank Steven G. Louie and Marvin Cohen for helpful discussions, and G.Y. McLean for experimental assistance. This work was supported by D.O.E. (Basic Energy Sciences, Materials Sciences Division, the $s p^{2}$ Materials Initiative). Nanotube production at Rice University was supported by the Office of Naval Research, the Robert A. Welch Foundation, and the Texas Advanced Technology Program.
${ }^{1}$ R. Martel, T. Schmidt, H. R. Shea, T. Hertel, and P. Avouris, Appl. Phys. Lett. 73, 2447 (1998).

${ }^{2}$ T. W. Odom, H. Jin-Lin, P. Kim, and C. M. Lieber, Nature (London) 391, 62 (1998).

${ }^{3}$ J. W. G. Wildoer, L. C. Venema, A. G. Rinzler, R. E. Smalley, and C. Dekker, Nature (London) 391, 59 (1998).

${ }^{4}$ S. J. Tans, R. M. Verschueren, and C. Dekker, Nature (London) 393, 49 (1998).

${ }^{5}$ A. M. Rao, P. C. Eklund, S. Bandow, A. Thess, and R. E. Smalley, Nature (London) 388, 257 (1997).

${ }^{6}$ R. S. Lee, H. J. Kim, J. E. Fischer, A. Thess, and R. E. Smalley, Nature (London) 388, 255 (1997).

${ }^{7}$ L. Grigorian, G. U. Sumanasekera, A. L. Loper, S. Fang, J. L. Allen, and P. C. Eklund, Phys. Rev. B 58, R4195 (1998).

${ }^{8}$ S. J. Tans, M. H. Devoret, H. Dai, A. Thess, R. E. Smalley, L. J.
Geerligs, and C. Dekker, Nature (London) 386, 474 (1997).

${ }^{9}$ D. H. Cobden, M. Bockrath, P. L. McEuen, A. G. Rinzler, and R. E. Smalley, Phys. Rev. Lett. 81, 681 (1998).

${ }^{10} \mathrm{We}$ note that none of the procedures described hereafter have a significant effect on metallic tubes, and thus we will focus exclusively on the properties of semiconducting tubes.

${ }^{11}$ We have never seen $n$-type behavior in undoped samples, even at large positive gate voltages. We believe that this is because in the back gated geometry the contact region is screened from the gate by the leads. Thus, the contact regions remain $p$-type regardless of the gate voltage, preventing the injection of charge into the $n$-type region.

${ }^{12}$ K. Esfarjani, A. A. Farajian, Y. Hashi, and Y. Kawazoe, Appl. Phys. Lett. 74, 79 (1999). 